\title{
Numerical Investigation of Flow around Two Tandem Identical Trapezoidal Cylinders
}

\author{
Yupu Wang $\mathbb{D},,^{1,2}$ Wenming Cheng $\mathbb{D}^{1,2}$ Run Du $\mathbb{D}^{1,2}$ Shubiao Wang $\mathbb{D}^{1,2}$ \\ and Yong Deng ${ }^{1,2}$ \\ ${ }^{1}$ School of Mechanical Engineering, Southwest Jiaotong University, Chengdu 610031, China \\ ${ }^{2}$ Technology and Equipment of Rail Transit Operation and Maintenance Key Laboratory of Sichuan Province, \\ Chengdu 610031, China
}

Correspondence should be addressed to Run Du; rdu@swjtu.edu.cn

Received 31 December 2019; Revised 28 February 2020; Accepted 11 March 2020; Published 27 April 2020

Academic Editor: Sébastien Poncet

Copyright (C) 2020 Yupu Wang et al. This is an open access article distributed under the Creative Commons Attribution License, which permits unrestricted use, distribution, and reproduction in any medium, provided the original work is properly cited.

The trapezoidal beam structure is ubiquitous in giant engineering equipment, while their aerodynamic characteristics have not been clearly understood. Numerical simulation method was adopted to investigate the flow around two tandem identical trapezoidal cylinders. The study was conducted using a Reynolds number of $2.2 \times 10^{4}$, and with a spacing ratio varying from 0.5 to 10 . The incompressible twodimensional finite volume method was used for solving Reynolds-Averaged Navier-Stokes (RANS) equations with realizable $k-\varepsilon$ model. The effects of cylinder geometry and spacing between the cylinders on aerodynamic characteristics, unsteady flow patterns, time-averaged flow characteristics, and flow instability was studied. The results show that the flow around the two tandem trapezoidal cylinders is highly dependent on the spacing ratio. The flow modes can be classified into: extended-body regime (Mode I, $S^{*} \leq 1$ ), reattachment regime (Mode II, $2 \leq S^{*} \leq 3$ ), and binary regime (Mode III, $S^{*} \geq 4$ ). We explored their respective flow characteristics and distinctions through the force/pressure coefficients, time-average streamwise velocity, and the flow field evolution.

\section{Introduction}

The flow over a bluff body is often accompanied by complicated phenomena such as flow separation, reattachment, and unsteady vortex shedding in the wake region. The repeated occurrence of large-scale vortex shedding repetition makes the structure susceptible to vibration, resulting in structural fatigue damage.

For a long time, scholars have focused on the flow problem of square, rectangular, or circular cross-section structures, but less attention has been paid to trapezoidal or other non-rectangular cross-sections. In the experiment, Luo et al. [1] investigated the influence of the afterbody shape and the angle of incidence on the flow structure around the prism by means of wind tunnel measurement and water tunnel flow field visualization. They found the main effect of the fluctuating aerodynamic forces differences is the proximity of the separated shear layers to their corresponding side faces. Venugopal et al. [2] carried out an experimental study on the influence of upstream disturbance and blockage on the flow around the trapezoid cylinder. Blockage refers to the ratio of the characteristic height of the bluff body to the flow field $(D / H)$. The results show that the blockage ratio of 0.3 is found to be the best among all the blockages $(0.14,0.24$, and 0.3$)$ under both disturbed and undisturbed conditions.

In numerical simulation, Lee [3] studied the flow around non-rectangular sharp-edged prisms (trapezoidal cylinder) at the Reynolds number from 25 to 1000 . The study shows that the structure of bluff bodies and Reynolds number have great influence on the characteristics of flow recirculation, flow separation, and flow interactions. Kahawita and Wang [4] studied the dynamic instability of a trapezoidal cylinder wake through two-dimensional numerical simulation. The spline method of fractional steps was used to study the Bénard-von Kármán hydrodynamic instability behind trapezoidal bluff body. Research found that the effect of trapezoid height seems to be the main effect on the value of Strouhal number, when compared with the effect of the 
smaller trapezoid bottom width. Cheng and Liu [5] studied the influence of rear-to-front width ratio $(\lambda)$ from 0 to 1 (section varied from triangle to square) on the flow characteristics. The results show that $\lambda=0.5$ makes the separation state of the shear layer and the wake vortex have the opposite trend. This means, when $\lambda<0.5$, the vortex shedding frequency increases with the increase of $\lambda$, but when $\lambda>0.5$, the frequency showed a negative correlation with the width. Study of Chung and Kang [6] showed that the Strouhal number of the trapezoidal cylinder depends on the Reynolds number and height ratio, which is the ratio of the height of the rear face to that of the front face $(d / D)$. Dhiman and Hasan [7] analyzed the flow separation and flow state of the trapezoidal cylinder under low Reynolds number $(\operatorname{Re}=1-150)$. Ding et al. [8] studied the flow induced vibration characteristics of the cylinder at high Reynolds number $\left(\operatorname{Re}=3 \times 10^{4} \sim 1.1 \times 10^{5}\right)$ and analyzed the amplitude, frequency, and vortex shape of the cylinder. Miau et al. [9] studied the low-frequency behavior of vortex shedding embedded in the trapezoid cylinder with the Reynolds number ranging from $7.9 \times 10^{3}$ to $3.24 \times 10^{4}$.

There has been much research on the flow past two tandem cylinders. Zdravkovich [10-12] conducted a series of studies on the effects of interference between cylinders and divided them into three basic flow interference categories based on the position of the downstream cylinder. The three categories they proposed are as follows. (1) Proximity interference: the free shear layers separated from the upstream cylinder do not reattach on the downstream cylinder, but form directly the vortex behind the downstream cylinder. (2) Wake interference: the free shear layer separated from the upstream cylinder reattaches to the downstream cylinder. This mode creates a vortex only behind the downstream cylinder. (3) Binary vortices: two vortex channels are formed behind the two cylinders. The separated shear layers of the upstream cylinder are rolled up alternately in front of the downstream cylinder and form vortices. The vortex street behind the downstream cylinder is called binary flow because each vortex street is actually composed of two vortices formed, respectively, from the upstream cylinder and the downstream cylinder, and the binary vortex causes excessive fluctuations in the downstream cylinder. Meneghini et al. [13] studied numerically the vortex shedding and flow interference between two cylinders in tandem and in side-byside arrangements. Jiang et al. [14] used the LBM (Lattice Boltzmann Method) to study the flow-induced vibration of one and two tandem circular cylinders and discussed the mechanism of vibration generation. Sumner [15] reviewed the understanding of the flow around two identical cylinders in a stable cross flow. The structure and behavior of nearwake and intermediate-wake of the two cylinders, as well as the general trends of the aerodynamic coefficient and Strouhal number, were summarized. The flow patterns can be roughly divided into (i) extended-body regime; (ii) reattachment regime; (iii) coshedding regime.

Sohankar and Etminan [16] numerically studied the flow characteristics and heat transfer on two equal square cylinders arranged in series at a low Reynolds number $(\mathrm{Re}=1 \sim 200)$. A floating stability analysis by Choi et al. [17] was performed to detect the occurrence of secondary instability in laminar flow around two identical square cylinders. The presence of adjacent cylinders can change the flow topology, resulting in changes in stability characteristics. Parametric studies were conducted with the gap between two square cylinders as the key parameter. Based on the gap, three different basic flow patterns were found and different modes of secondary instability were identified for each basic flow pattern. Yen et al. [18] used the PIV (Particle Image Velocimetry) and flow topology method to investigate the two squares cylinders in tandem experimentally. They divided flow characteristics into three modes: single mode, reattached mode, and binary mode at low Reynolds number $(\mathrm{Re}=535)$. Some researchers carried out studies on the flow around two cylinders, and one of them (upstream/downstream) was forced to oscillate in the lateral direction. $\mathrm{Xu}$ and Zhou [19] used a hot-wire anemometer to study a series of square columns with Reynolds number $\mathrm{Re}=800 \sim 4.2 \times 10^{4}$ and cylinder center-to-center distance $L / d=1-15$. They proposed four distinct St-Re behavior modes. More et al. [20] used PIV, hot-wire anemometers, and flow visualization techniques to study the effect of the spacing between two series-connected cylinders and the vibration of the upstream cylinder on the flow characteristics. The experiments were performed at Reynolds number of 295 . The study also captured three different flow patterns similar to previous studies.

To sum up, scholars have made some achievements in the study of the flow around a single trapezoidal cylinder, but the investigation on the flow around two tandem trapezoidal cylinders is still insufficient. In view of this, this paper numerically simulated the flow around two tandem trapezoidal cylinders at $\mathrm{Re}=2.2 \times 10^{4}$. We studied the aerodynamic characteristics, flow regime, and development pattern of the tail vortex at different spacing ratios so as to provide reference for the structural design of engineering equipment.

\section{Numerical Details and the Governing Equations}

2.1. Problem Description. The description of the problem is shown in Figure 1. Two identical trapezoidal cylinders in tandem were exposed to a constant free stream with uniform velocity $U(=1.61 \mathrm{~m} / \mathrm{s})$. The flow was described in a Cartesian coordinate system ( $x$ and $y$ ), where the $x$-axis was aligned with the inlet flow direction and the $y$-axis was perpendicular to the $x$-axis. The origin was located at the center point of the upstream cylinder. The height $D$ on the windward side of the trapezoidal cylinder was equal to $200 \mathrm{~mm}$. All dimensions of the computational domain refer to the height of the windward side of trapezoidal cylinder (D). The width $B$ of the trapezoidal cylinder was equal to $D$, and the height $d$-the rear face of the trapezoidal cylinder was equal to $0.5 D$.

In the $y$-direction, the vertical distance between the upper and lower boundaries of the domain was $H(=20 D)$, which determines the blockage rate of the flow field. The distance from the center of the upstream cylinder to the inlet 


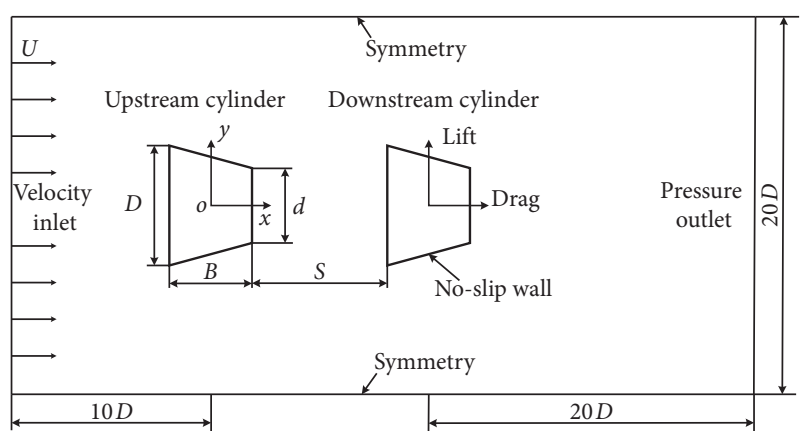

FIGURE 1: The geometry of flow domain and boundary conditions.

was $10 D$, and the distance from the center of the downstream cylinder to the outlet was $20 D$. The spacing ratio $S^{*}$ was defined as $S^{*}=S / D$, where $S$ represents the distance between the rear face of the upstream cylinder and the front face of the downstream cylinder. $S^{*}$ was selected as $0.5,1,2$, $3,4,6$, and 10 .

2.2. Numerical Calculation Governing Equations. Based on continuum theory and Newton's second law, the NavierStokes equation of fluid motion can be derived. Due to the presence of turbulent flow, the Navier-Stokes equation is highly nonlinear and it is difficult to obtain a theoretical solution. In the RANS (Reynolds-Averaged Navier-Stokes) equations, the variables are decomposed into the mean and fluctuating components. For the incompressible flow in this paper, the RANS equations can be written as follows:

$$
\begin{gathered}
\frac{\partial \bar{u}_{i}}{\partial x_{i}}=0 \\
\rho \frac{\partial \bar{u}_{i}}{\partial t}+\rho \frac{\partial \bar{u}_{j} \bar{u}_{i}}{\partial x_{j}}=-\frac{\partial \bar{p}}{\partial x_{i}}+\frac{\partial}{\partial x_{j}}\left(2 \mu \bar{S}_{i j}-\rho \overline{u_{i}^{\prime} u_{j}^{\prime}}\right),
\end{gathered}
$$

where $\bar{S}_{i j}$ is strain rate tensor, $\bar{S}_{i j}=(1 / 2)\left(\left(\partial \bar{u}_{i} / \partial x_{j}\right)\right.$ $\left.+\left(\partial \bar{u}_{j} / \partial x_{i}\right)\right)$.

Therefore, to make the equation closure, an effective method which employs Boussinesq hypothesis [21] to relate the Reynolds stresses to the mean velocity gradients is introduced:

$$
-\rho \overline{u_{i}^{\prime} u_{j}^{\prime}}=\mu_{t}\left(\frac{\partial \bar{u}_{i}}{\partial x_{j}}+\frac{\partial \bar{u}_{j}}{\partial x_{i}}\right) .
$$

Then, how to determine the turbulent viscosity $\mu_{t}$ becomes a key to the solution of problems in calculation. Shih et al. [22] proposed that the calculation of the turbulent viscosity of turbulent flow is directly related to the strain rate, and the turbulent viscosity can be expressed as

$$
\begin{aligned}
\mu_{t} & =\rho C_{\mu} \frac{k^{2}}{\varepsilon}, \\
\varepsilon & =\frac{\mu}{\rho} \overline{\left(\frac{\partial u_{j}^{\prime}}{\partial x_{j}}\right)^{2}},
\end{aligned}
$$

where $C_{\mu}$ can be defined as the standard value of 0.09 in the equilibrium boundary layer, and 0.05 in a strong homogeneous shear flow.

Based on the Boussinesq hypothesis, $\mu_{t}$ is an isotropic scalar quantity. Although this hypothesis is not strictly validated. On many occasions, models based on the Boussinesq hypothesis perform very well. The realizable $k-\varepsilon$ model has shown substantial improvements over the standard $k-\varepsilon$ model. Because of the flow in this paper is thermostatic and incompressible, the transport equations for $k$ and $\varepsilon$ in the realizable $k-\varepsilon$ model are

$$
\begin{aligned}
\frac{\partial}{\partial t}(\rho k)+\frac{\partial}{\partial x_{j}}\left(\rho k u_{j}\right)= & \frac{\partial}{\partial x_{j}}\left[\left(\mu+\frac{\mu_{t}}{\sigma_{k}}\right) \frac{\partial k}{\partial x_{j}}\right]+G_{k}-\rho \varepsilon \\
\frac{\partial}{\partial t}(\rho \varepsilon)+\frac{\partial}{\partial x_{j}}\left(\rho \varepsilon u_{j}\right)= & \frac{\partial}{\partial x_{j}}\left[\left(\mu+\frac{\mu_{t}}{\sigma \varepsilon}\right) \frac{\partial \varepsilon}{\partial x_{j}}\right]-\rho C_{1} S \varepsilon \\
& -\rho C_{2} \frac{\varepsilon^{2}}{k+\sqrt{v \varepsilon}}
\end{aligned}
$$

where

$$
\begin{aligned}
G_{k} & =\mu_{t} S^{2}, \\
C_{1} & =\max \left[0.43, \frac{\eta}{\eta+5}\right], \\
\eta & =S \frac{k}{\varepsilon}, \\
S & =\sqrt{2 S_{i j} S_{i j}}, \\
C_{2} & =1.9 .
\end{aligned}
$$

2.3. Calculation Area and Boundary Conditions. The calculation of flow area and boundary conditions are shown in Figure 1 . The tandem trapezoidal cylinders in this study were placed in a two-dimensional, unsteady, viscous, and incompressible flow. The boundary of the flow field should be sufficiently far from the cylinders to achieve a lower blockage rate to meet the requirement of free-stream conditions, and the blockage of the simulation examples in this paper was 0.05. The calculation boundary conditions for the flow field were set as follows:

Reynolds number: $2.2 \times 10^{4}$

Uniform velocity inlet: $u_{i}=U, u_{j}=0$

Pressure outlet: $0 \mathrm{~Pa}$

No-slip wall on the surface of the trapezoidal cylinder

Top and bottom boundaries: symmetry

Turbulence intensity of the flow: 5\% 
2.4. Definitions of Characteristic Quantities. The non-dimensional drag, lift, and pressure coefficient of the cylinder are defined as

$$
\begin{gathered}
C_{d}=\frac{F_{d}}{1 / 2 \rho U^{2}}, \\
C_{l}=\frac{F_{l}}{1 / 2 \rho U^{2}}, \\
C_{p}=\frac{p-p_{\infty}}{1 / 2 \rho U^{2}} .
\end{gathered}
$$

The formula for the Reynolds and Strouhal number is

$$
\begin{aligned}
\operatorname{Re} & =\frac{U D}{v}, \\
\mathrm{St} & =\frac{f D}{U} .
\end{aligned}
$$

The time average and root mean square of the force coefficient fluctuation are calculated as follows (take $C_{d}$ as an example):

$$
\begin{aligned}
C_{d, \mathrm{avg}} & =\frac{\int_{0}^{T} C_{d}(t) \mathrm{d} t}{T}, \\
C_{d, \mathrm{rms}} & =\sqrt{\frac{\int_{0}^{T}\left[C_{d}(t)-C_{d, \mathrm{avg}}\right]^{2} \mathrm{~d} t}{T}},
\end{aligned}
$$

where $T$ is the fluctuation period.

2.5. Code Validation and Grid Independence Study. Figure 2 shows the non-uniform grid for the tandem trapezoid. In order to ensure the accuracy of the numerical simulation, the flow around a square and around a trapezoidal cylinder at $\operatorname{Re}=2.2 \times 10^{4}$ was calculated first. The calculation area was divided based on orthogonal grids, and the thickness of the first grid layer near the cylinder surface $(\Delta d)$ was $0.0213 D$ and $0.0355 D$, respectively. The Courant number (Co) is used to adjust the stability and convergence of the calculation. It is defined as follows:

$$
\mathrm{Co}=\frac{U \Delta t}{D \Delta d} \text {. }
$$

According to the criterion that the Courant number is less than 1 , the time steps were $1.33 \times 10^{-3} \mathrm{~s}$ and $2.12 \times 10^{-3} \mathrm{~s}$, respectively. The calculation used the PISO algorithm, and the convergence value of the continuity equation was set to $10^{-5}$. Numerical results are shown in Table 1.

The drag coefficient for the calculation of the flow around the square-cylinder measured in this study was consistent with the experimental results of Lyn et al. [23] and Norberg [24]. At the same time, the calculation results matched well with the simulation results of Sohankar [25], Srinivas et al. [26], and Zhou et al. [27]. The Strouhal number of the square cylinder measured in the present study accord with the research findings of Atsushi and Okajima [28]. The

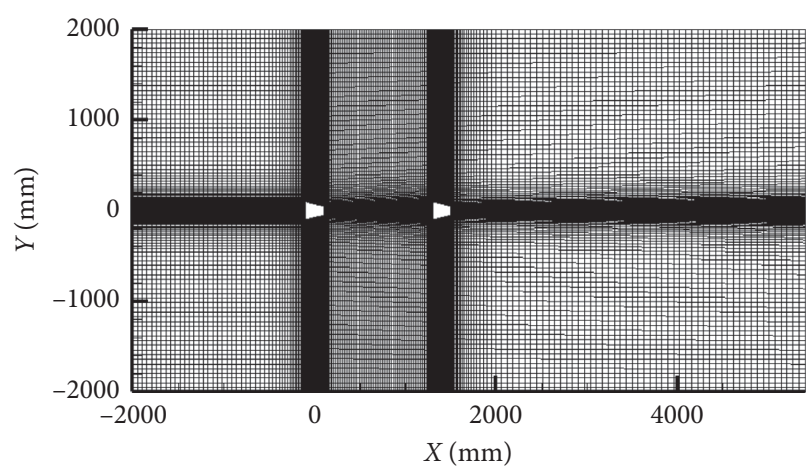

FIGURE 2: Non-uniform grid for the tandem trapezoidal cylinders.

time average drag coefficients $C_{d \text {,avg }}$ and St of trapezoidal cylinders were in good agreement with the experimental values of Luo et al. [1], and the difference was less than $10 \%$. It can be seen that the numerical simulation performed in this paper is accurate and reliable.

In both cases, grid thickness of the first layer near the cylinder surface was less than $3.6 \%$, which was consistent with the literature value. Considering the computational cost of the calculation example, grid thickness of the first layer was determined as $\Delta d=0.0355 D$ in this paper for subsequent calculations.

\section{Results and Discussion}

3.1. Drag and Lift Coefficients. For the case of blunt body flow in engineering, the average drag coefficient $\left(C_{d \text {,avg }}\right)$ can be used to measure the wind load on bridges, buildings, or mechanical structures. The lift oscillation also influences safety in engineering. Figure 3 shows the variation of the average drag coefficient $\left(C_{d \text {,avg }}\right)$ and root-mean-square lift coefficient $\left(C_{l, \mathrm{rms}}\right)$ for the two tandem trapezoidal cylinders at different spacing ratios $S^{*}$.

In Figure 3(a), compared with the average drag coefficient of a single trapezoidal cylinder [1], the $C_{d \text {,avg }}$ of the upstream cylinder was slightly reduced when $S^{*}=0.5,1$. As the $S^{*}$ increased, the $C_{d \text {,avg }}$ of the upstream trapezoidal cylinder remained basically unchanged and was similar to that of a single one. The $C_{d \text {,avg }}$ of the downstream trapezoidal cylinder was smaller than that of the single one due to the influence by the wake of the upstream cylinder. The value of $C_{d \text {,avg }}$ were negative when $S^{*}=0.5,1$. When the spacing ratio increased to $S^{*}=2$, the $C_{d \text {,avg }}$ changed from negative to positive and maintained at a relatively high value $\left(S^{*}=2,3\right)$. As the spacing ratio continued to increase $\left(S^{*} \geq 4\right)$, the $C_{d \text {,avg }}$ of the downstream trapezoidal cylinder first decreased and then gradually remained unchanged. Referring to study of Yen et al. [18] on tandem square cylinders, we divided the flow modes around two identical tandem trapezoidal cylinders into three categories: extended-body regime (Mode I, $S^{*} \leq 1$ ), reattachment regime (Mode II, $2 \leq S^{*} \leq 3$ ), and binary regime (Mode II $S^{*} \geq 4$ ). The distinction between mode I and mode II was a positive-negative conversion of the average drag coefficient of the downstream trapezoidal cylinder. 
TABLE 1: Comparison of integral parameters.

\begin{tabular}{|c|c|c|c|c|c|c|c|c|}
\hline Examples & $\Delta d / D$ & $\operatorname{Re}\left(\times 10^{3}\right)$ & $C_{d, \text { avg }}$ & St & $C_{d, \mathrm{tms}}$ & $C_{l, \mathrm{rms}}$ & $C_{\mathrm{pb}}$ & $L_{r}$ \\
\hline \multirow{2}{*}{ Square cylinder } & 0.0213 & 22 & 2.11 & 0.132 & 0.25 & 1.35 & -1.68 & 1.31 \\
\hline & 0.0355 & 22 & 2.09 & 0.133 & 0.24 & 1.39 & -1.62 & 1.33 \\
\hline \multirow{2}{*}{ Trapezoidal cylinder } & 0.0213 & 22 & 2.62 & 0.143 & 0.20 & 1.62 & -1.70 & 1.23 \\
\hline & 0.0355 & 22 & 2.59 & 0.140 & 0.20 & 1.61 & -1.68 & 1.28 \\
\hline \multicolumn{2}{|c|}{ Lyn et al. [23] (Exp) } & 21.4 & 2.10 & 0.130 & - & - & -1.60 & 1.38 \\
\hline \multicolumn{2}{|c|}{ Norberg [24] (Exp) } & 22 & 2.10 & 0.130 & - & - & -1.37 & - \\
\hline \multicolumn{2}{|c|}{ Sohankar [25] (LES) } & 22 & 2.32 & 0.132 & 0.20 & 1.54 & -1.63 & - \\
\hline \multicolumn{2}{|c|}{ Srinivas et al. [26] (LES) } & 21.4 & 2.03 & 0.130 & 0.18 & 1.29 & - & 1.31 \\
\hline \multicolumn{2}{|c|}{ Zhou et al. [27] (LES) } & 22 & 2.27 & 0.122 & 0.29 & 1.40 & -1.61 & 1.37 \\
\hline \multicolumn{2}{|c|}{ Atsushi and Okajima [28] (Exp) } & 20 & - & 0.133 & - & - & - & - \\
\hline \multicolumn{2}{|c|}{ Luo et al. [1] (Exp) } & 34 & 2.51 & 0.140 & - & - & -1.87 & 1.26 \\
\hline
\end{tabular}

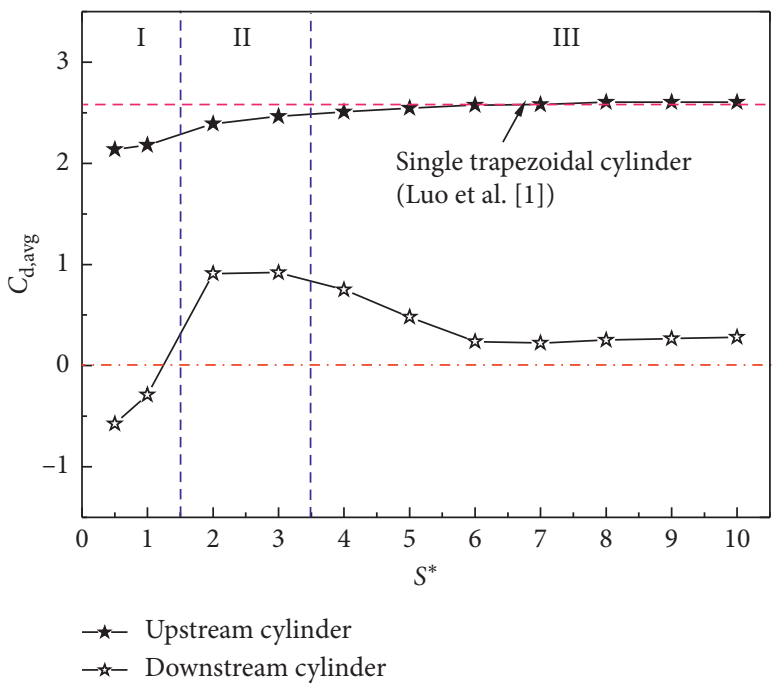

(a)

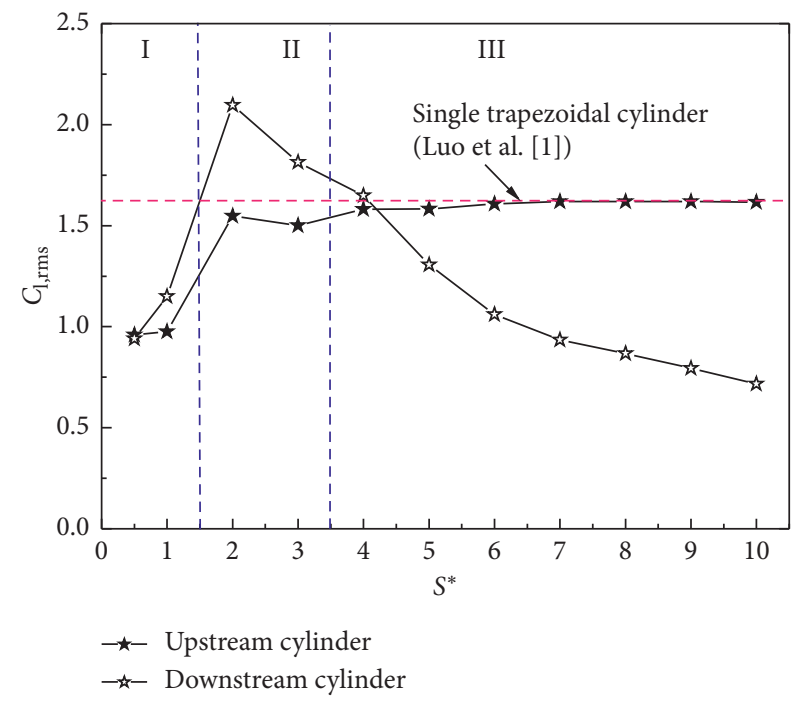

(b)

Figure 3: Variation of dynamic force coefficients $\left(C_{d \text {,avg }}, C_{l, \text { rms }}\right)$ with $S^{*}$ for the two tandem trapezoidal cylinders. (a) Average drag coefficient (b) Root-mean-square lift coefficient.

From Figure 3(b), the $C_{l, \mathrm{rms}}$ of the upstream trapezoidal cylinder maintained a low value in mode I, while it remained basically unchanged in mode II and mode III. The value of $C_{l, \mathrm{rms}}$ was equal to that of a single cylinder [1] in mode II and mode III. This shows that when $S^{*} \geq 2$, the upstream trapezoidal cylinder was less affected by the downstream trapezoidal cylinder. In mode I, the $C_{l, \mathrm{rms}}$ of the upstream and downstream trapezoidal cylinders were close. The $C_{l, \mathrm{rms}}$ of the downstream trapezoidal cylinder had a large rise from $S^{*}=1$ to $S^{*}=2$, and then reduced gradually with the increase of the spacing ratio $\left(S^{*} \geq 2\right)$. This meant that the effect of the upstream trapezoidal cylinder on the downstream trapezoidal cylinder gradually weakened as the spacing ratio increased. The $C_{l, \text { rms }}$ of the downstream trapezoidal cylinder was larger than that of the upstream trapezoidal cylinder in mode II and reversed in mode III. Compared with the $C_{l, \mathrm{rms}}$ of a single trapezoidal cylinder, the results of mode I and mode III were smaller, while the results of mode II were larger.

3.2. Vortex Frequency and Strouhal Number. The lift force spectrogram of the two tandem trapezoidal cylinders with different spacing ratios are shown in Figure 4. The lift force spectrogram was related to the vortex frequency and Strouhal number of the flow field. For the upstream cylinder, the lift force oscillation was relatively stable at $S^{*}=0.5$, which means the interference between the two trapezoidal cylinders was weak. When $2 \leq S^{*} \leq 4$, the spectrogram changed drastically. As the spacing ratio increased, the interference between the two cylinders gradually disappeared, and the oscillation became stable again.

For the downstream cylinder, its lift force spectrogram had the same trend as the upstream cylinder. The difference was that when the pitch ratio was $2 \leq S^{*} \leq 4$, the vibration of the downstream cylinder was more severe. This phenomenon occurs because the vortex generated by the upstream cylinder directly acts on the downstream cylinder.

Vortex frequency and Strouhal number (St) for different spacing ratios are shown in Figure 5, as referring to the study by Wang et al. [29] and Kim et al. [30] on the tandem square cylinders. According to the research of the variation of St with $S^{*}$ on the two tandem square cylinders, there is a dip of St with a minimum value of $S^{*} \approx 3$. As the $S^{*}$ increases, the 


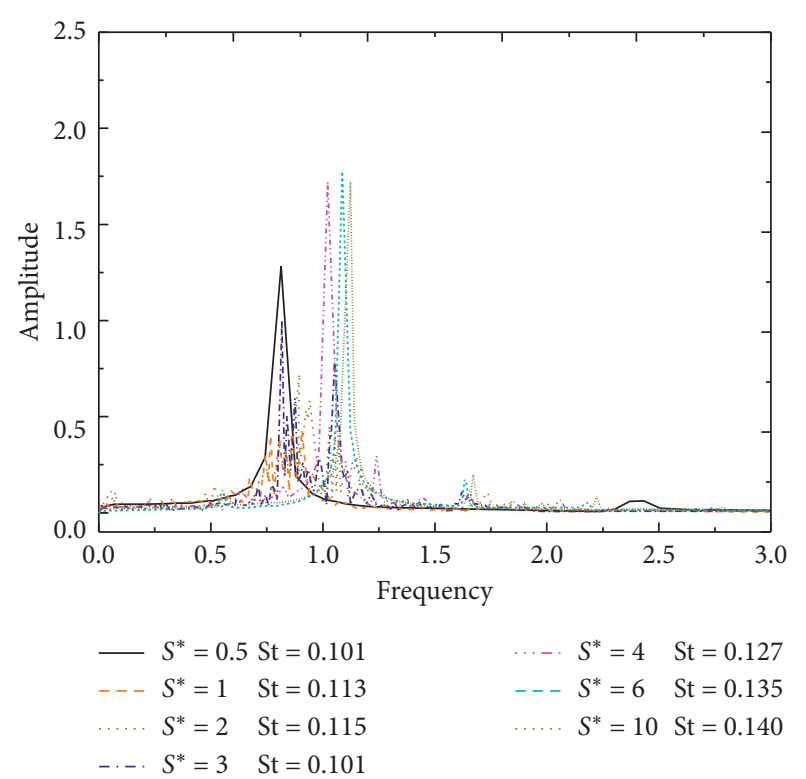

(a)

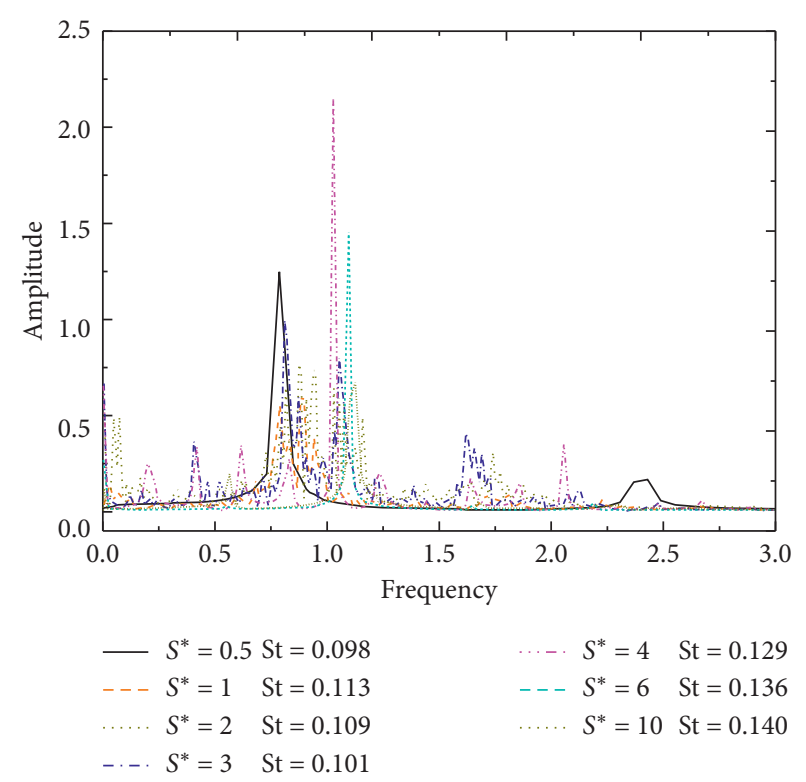

(b)

FIgURE 4: Lift force spectrogram under different spacing ratios. (a) Upstream and (b) downstream.

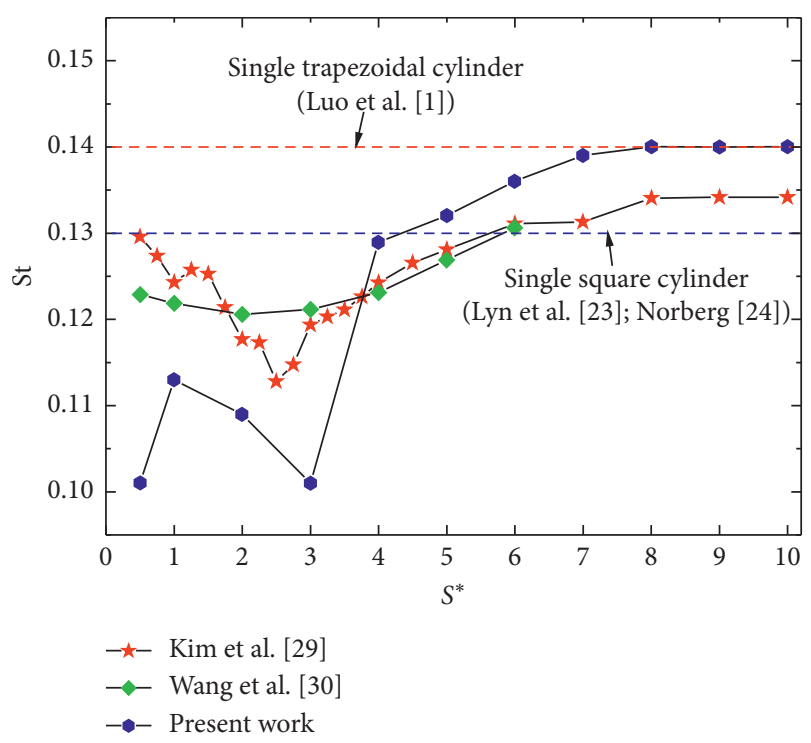

Figure 5: Variation of the Strouhal number (St) with $S^{*}$ for tandem cylinders.

interaction between the two cylinders weakens, and the value of $S t$ is close to that of the single cylinder $[23,24]$.

When the spacing was small, the St of the two tandem trapezoidal cylinders reduced first and then enlarged with the increase of the space ratio, which was similar to the finding of the study on the tandem square cylinders. When $S^{*} \geq 8$, the St was equal to the finding, in the case of single trapezoidal cylinder by Luo et al. [1], studied. This shows that, in the case of large spacing, two cylinders exhibited an independent mode like a single cylinder (Mode III).
3.3. Aerodynamic and Flow Characteristics. Figure 6(a) shows the time-averaged surface pressure coefficient $\left(C_{p}\right)$ around the upstream trapezoidal cylinder for different spacing ratios and the comparison with the single trapezoid. The $C_{p}$ on the windward side $\mathrm{AB}$ of the upstream trapezoidal cylinder was basically not affected by the spacing ratio, and the $C_{p}$ of the upper face $\mathrm{BC}$ and the rear face CD were greatly affected by the spacing ratio. The time-averaged surface pressure coefficient distribution trend was similar to the study results of the trapezoidal cylinder by Venugopal et al. [2] and Kahawita and Wang [4].

When the spacing ratio was relatively small $\left(S^{*} \leq 1\right)$, the pressure coefficient of the CD surface was affected by the reaction of the downstream cylinder, which resulted in a higher value. This result also explained why the $C_{d \text {,avg }}$ of the upstream cylinder was slightly reduced in model I, as shown in Figure 3. With the increase of the spacing ratio, the circumferential distribution of the upstream trapezoidal cylinder approached to that of the single one. When $S^{*}=0.5$, $1,3,4,6$, and 10 , the $C_{\mathrm{pb}}$ (pressure coefficient at point $\mathrm{D}$ ) was $-1.02,-1.22,-1.25,-1.50,-1.61$, and -1.64 , respectively. This meant that the effect of the downstream trapezoidal cylinder on the upstream one gradually decreased as the spacing ratio increased, as shown in Figure 3.

As Figure 6(b) shows, the circumferential pressure distribution of the downstream trapezoidal cylinder varied greatly under different spacing ratios. When the spacing ratio was very small $\left(S^{*} \leq 1\right)$, the $C_{p}$ on the windward side (EF) of downstream trapezoidal cylinder was negative, which lead to a negative $C_{d \text {,avg }}$ of the downstream cylinder. The upper face (FG) of the downstream trapezoidal cylinder was little affected by the change of spacing ratio, and it was basically around -0.7 . However, when $S^{*}=3$, the negative pressure of the FG face increased significantly, and the $C_{p}$ 


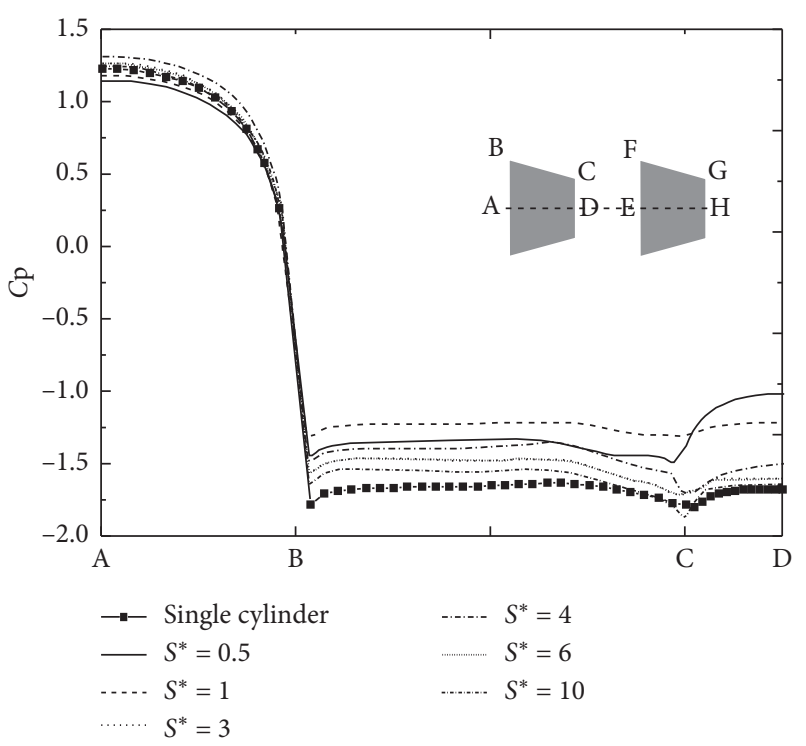

(a)

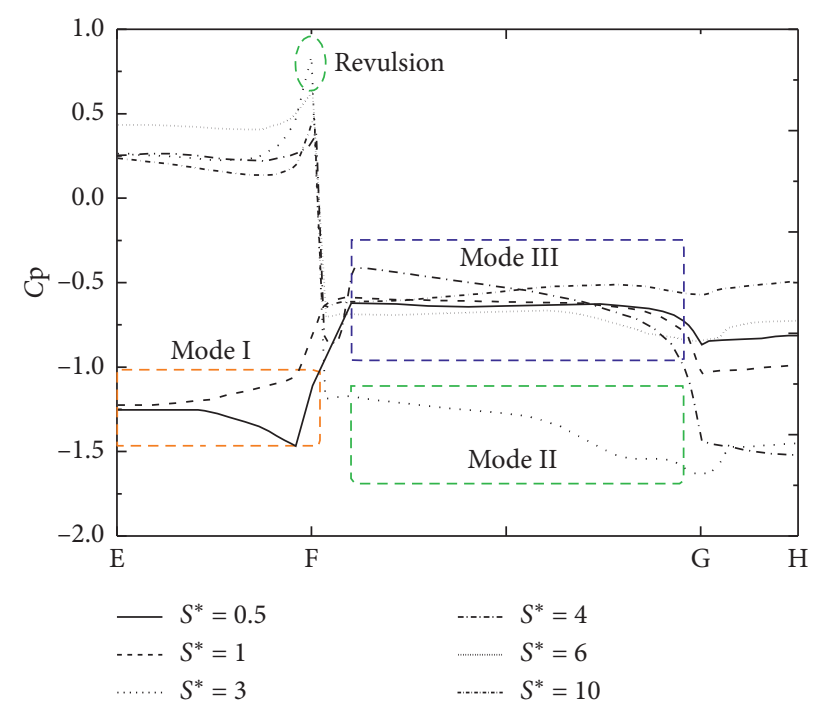

(b)

FiguRE 6: Time averaged pressure coefficient distribution around the trapezoidal cylinders for different spacing ratios. (a) Upstream cylinder and (b) downstream cylinder.

increased sharply around point F. This was due to the fact that the boundary layer separated from upstream cylinder reattached around point $\mathrm{F}$. When $S^{*}=3,4,6$, and 10 , the $C_{\mathrm{pb}}$ (pressure coefficient at point $\mathrm{H}$ ) was $-1.45,-1.52,-0.73$, and -0.50 , respectively, which meant the vortex in the wake region gradually moved away from the cylinder.

Figure 7(a) presents the time-averaged streamwise velocity distribution along the wake centerline, as shown in Figure 6(a), between the two trapezoidal cylinders. Velocity stagnation at points $\mathrm{D}$ and $\mathrm{E}$ was 0 . For smaller spacing ratios $\left(S^{*}=0.5,1\right)$, the time-average streamwise velocity was U-shaped, which meant that there was no vortex forming between the two cylinders. As the spacing ratio increases, the line shape changed to "S." The shape of time-average streamwise velocity distribution is similar to the result in the case of tandem circular and square cylinders studied by Zhang et al. [31] There was a stationary point (X) where the velocity changed from negative to positive. The stationary point in the fluid can indicate the position of vortex. At the spacing ratios $S^{*}=2,3$, the point $\mathrm{X}$ appeared near $x / D=1$. As the spacing ratio increased, the point $\mathrm{X}$ moved forward and stabilized around $x / D=0.6$. In summary, as the spacing ratio increased, the wake flow field of the upstream cylinder changed from no vortices, to distant vortices $(x / D \approx 1)$ and closer vortices $(x / D \approx 0.6)$.

Figure 7(b) shows the streamwise velocity distribution at the rear of the downstream trapezoidal cylinder. When the spacing ratio $S^{*}=0.5,1$, the time-average streamwise velocity of the downstream had a similar shape. As the spacing ratio increased, the downstream trapezoidal cylinder entered into mode II and mode III, and the wake speed decreased due to the influence of the upstream cylinder.

It can be seen from Figure 8 that the pulsating velocity $\left(u_{i, \mathrm{rms}}\right)$ in the wake direction and vertical pulsating velocity $\left(u_{j, \mathrm{rms}}\right)$ gradually decreased as the interval ratio $S^{*}$ increased.
The number of wave crests was related to the generation of vortices. At the spacing ratio of $S^{*}=4$, the RMS velocity of the downstream appeared to be an asymmetric peak $(y<0)$. It can be inferred that there were asymmetric vortices. When $S^{*}=10$, the double peaks show that there was a double-row tail vortex, as shown in Figure 9.

3.4. Flow Field Evolution. Figure 9 gives the instantaneous flow visualization images obtained during the evolution of flow field around two tandem trapezoidal cylinders. The three spacing ratios in Figure 9 represented the flow field characteristics in mode I $\left(S^{*}=0.5\right)$, mode II $\left(S^{*}=3\right)$, and mode III $\left(S^{*}=6\right)$, respectively. When $S^{*}=0.5$, the downstream trapezoidal cylinder was located in the trailing vortex formed by the boundary layer separation from the upstream cylinder (Figure 9(a)). The two trapezoidal cylinders behaved as a whole cylinder in the flow field (Figure 9(b)). Hence, model I was called an extended-body regime. In this mode, the wake was a single-row vortex (Figure 9(c)). The phenomenon was similar to the single trapezoidal cylinder.

When the spacing ratio increases to $S^{*}=3$, the boundary layer separated from the upstream trapezoidal cylinder reattach on the front or upper face of the downstream trapezoidal cylinder (Figure 9(a)). The tail of the upstream trapezoidal cylinder formed a slightly backward vortex (compare to the tail image of $S^{*}=6$ in Figure 9(a)). The boundary layer flow, attaching to the downstream trapezoidal cylinder, made the wake disorder (Figure 9(b)). This also explained the reason for the high $C_{l, \text { rms }}$ of the downstream trapezoidal cylinder in mode II in Figure 3. In this mode, a double-row vortex street appeared in the wake of the downstream trapezoidal cylinder (Figure 9(c)).

When $S^{*}=6$, the wake vortex of the upstream trapezoidal cylinder was formed. Under these circumstances, the 


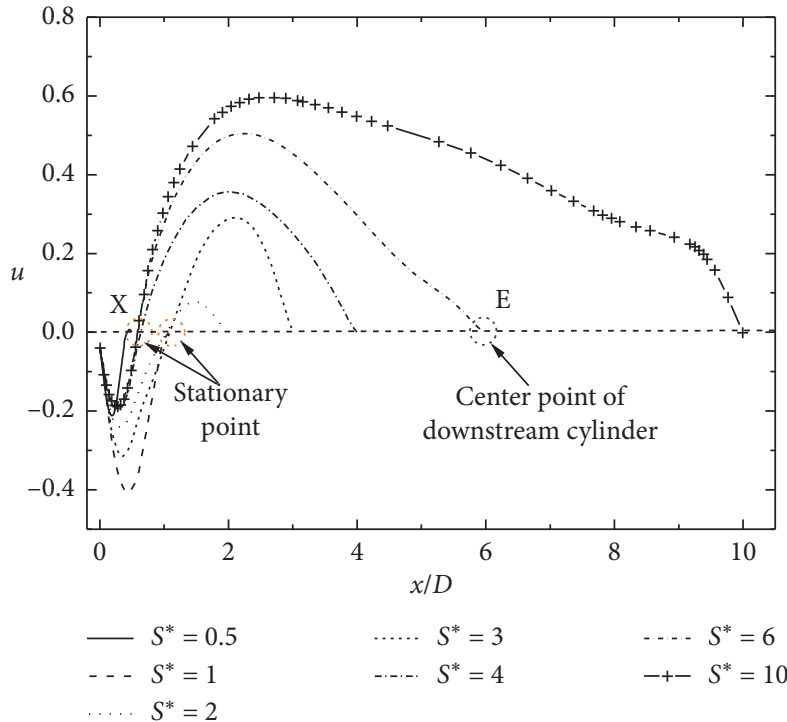

(a)

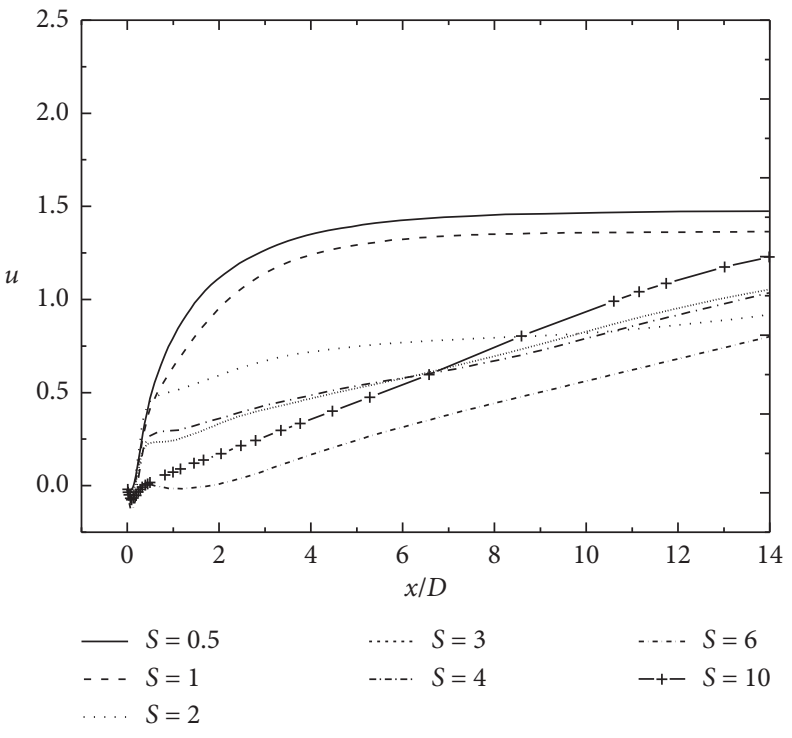

(b)

FIGURE 7: Distribution of time-average streamwise velocity along the wake centerline $(y=0)$. (a) The space between the two trapezoidal cylinders and (b) downstream cylinder wake region.
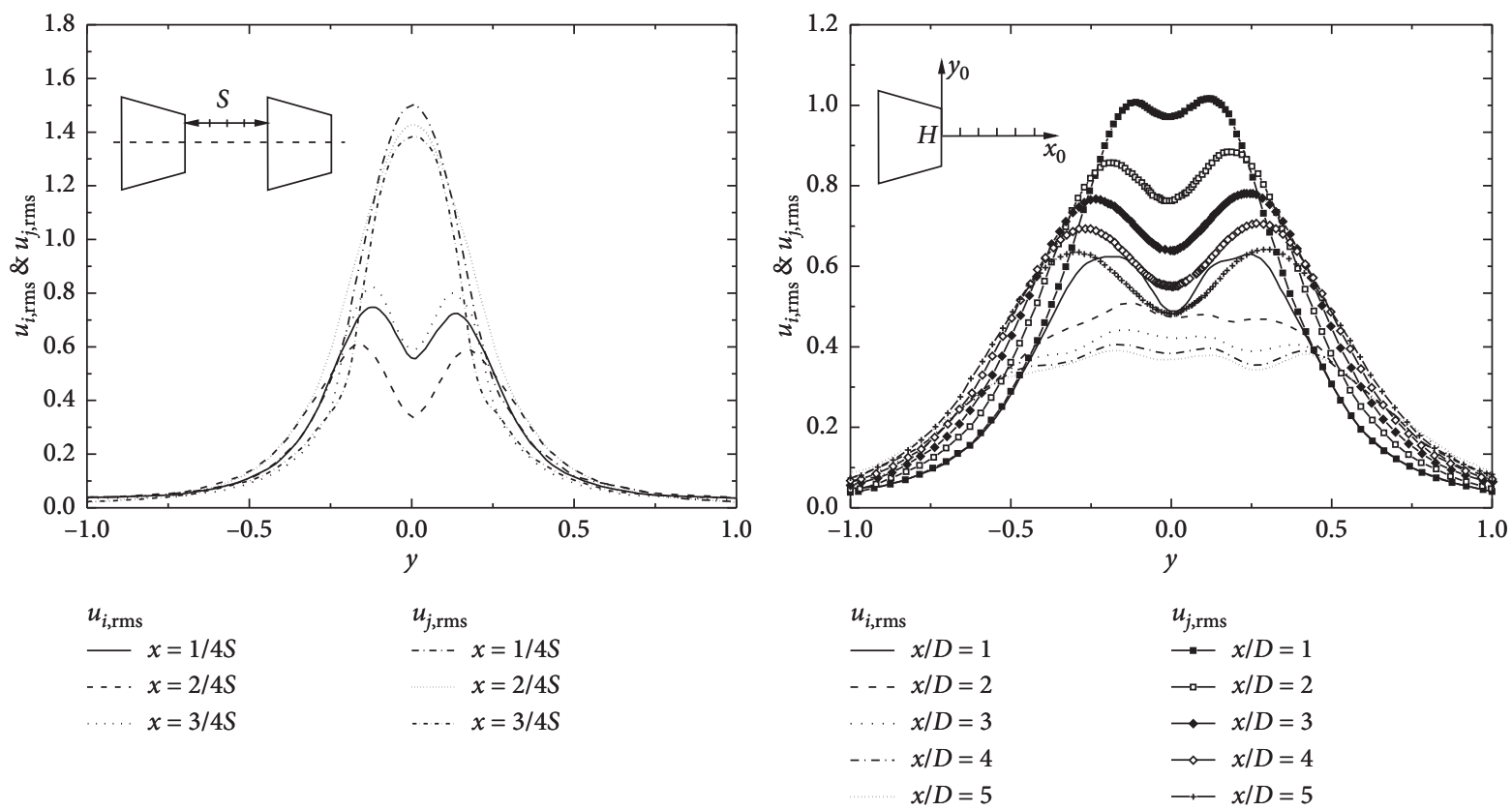

(a)

Figure 8: Continued. 

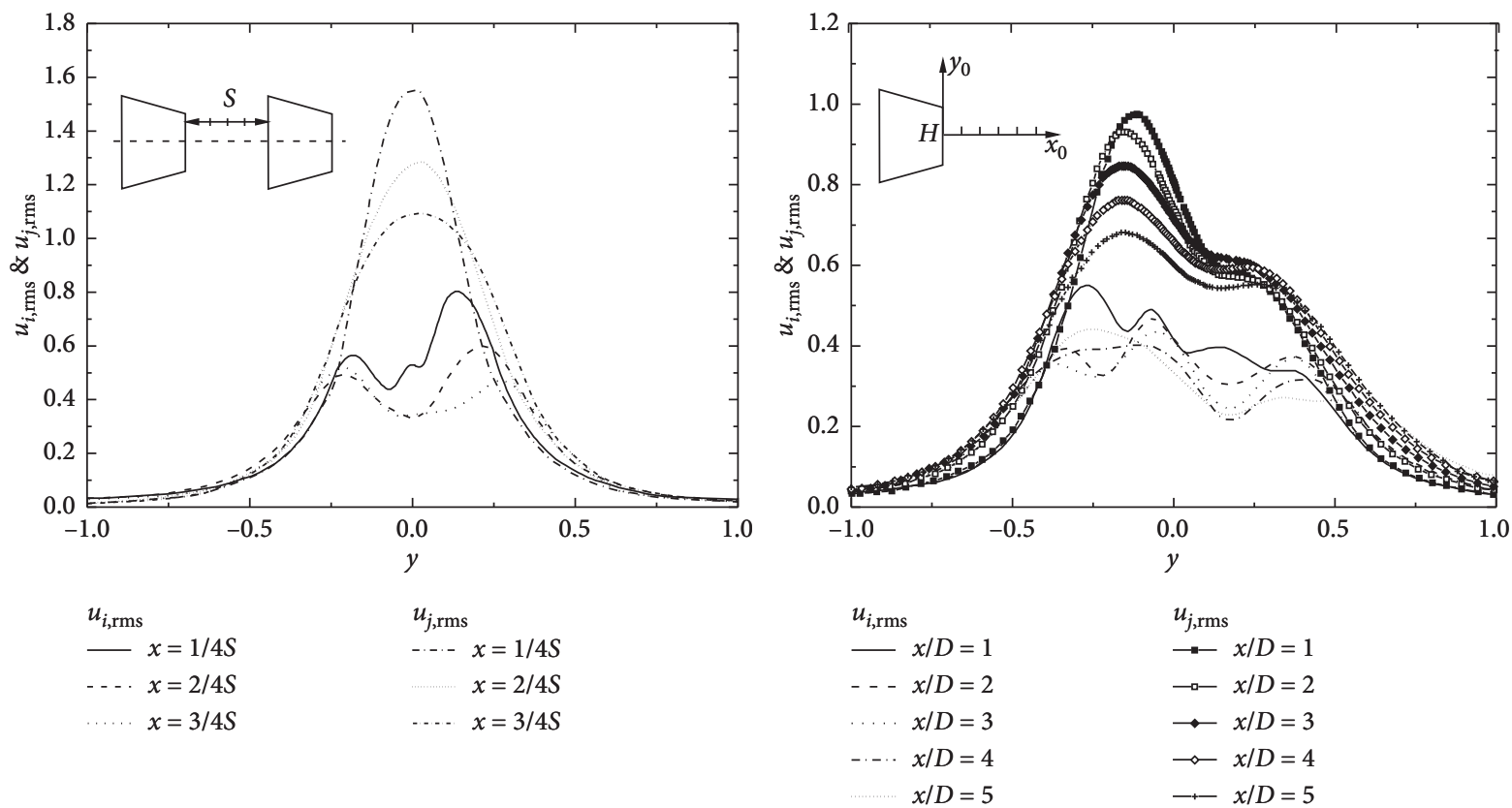

(b)
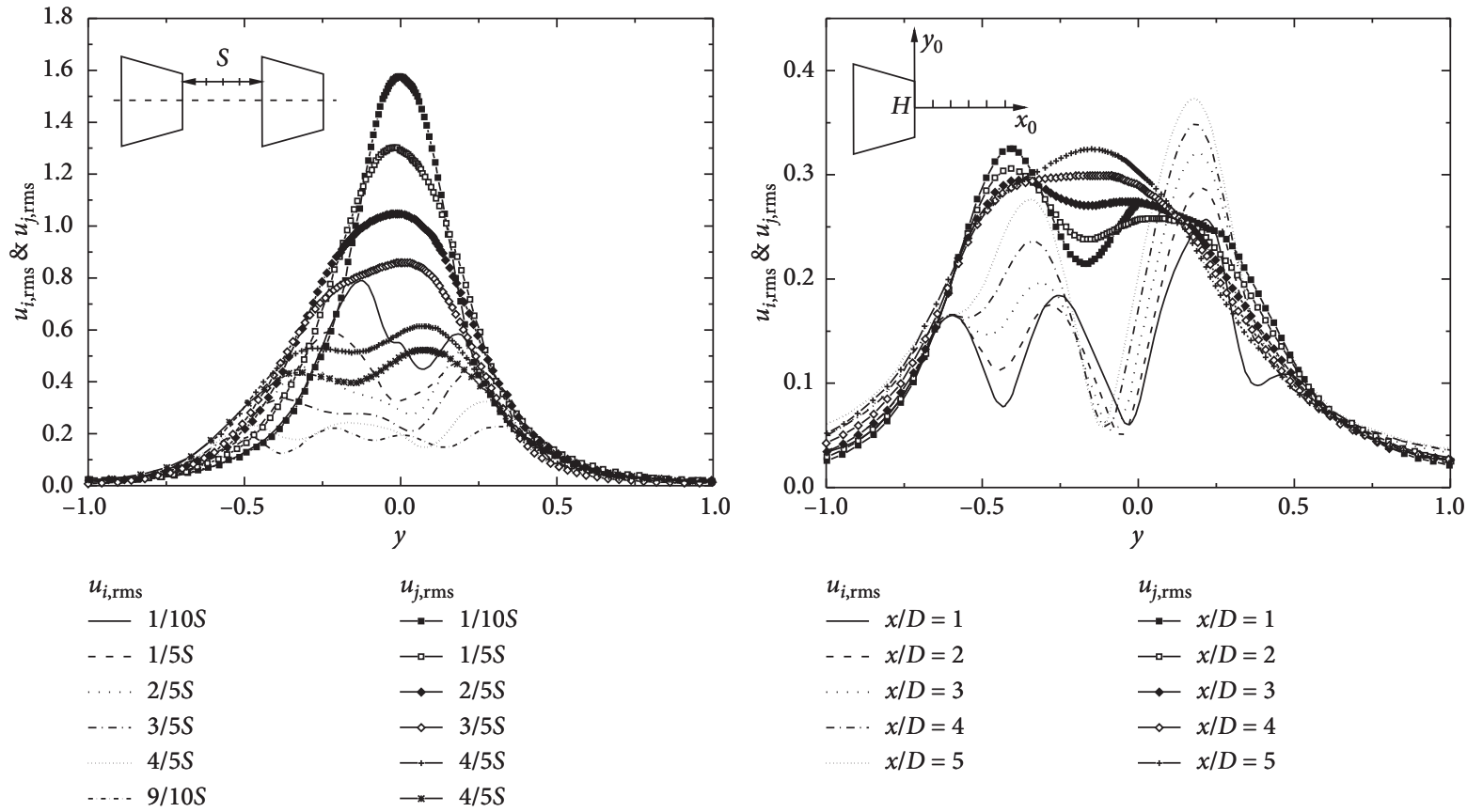

(c)

FIGURE 8: Distribution of RMS velocity $\left(u_{i, \mathrm{rms}} \& u_{j, \mathrm{rms}}\right)$ along the wake centerline with different spacing ratios: (a) $S^{*}=2$; (b) $S^{*}=4$; (c) $S^{*}=10$.

flow around the upstream trapezoidal cylinder was similar to that around single one, and it was hardly affected by the downstream trapezoidal cylinder (Figure 9(a)). The downstream trapezoidal cylinder was still influenced by the upstream cylinder, appearing to be a double-row tail vortex.

3.5. Characteristics and Distinction of the Three Modes. Great attention has been paid to the flow around two tandem cylinders in the literature. The studies of the flow around two tandem circular cylinders at different Reynolds numbers and with different spacing ratios were conducted by Zdravkovich [10-12], Sumner [15], and Xu and Zhou [19]. Zdravkovich [10] classified the flow around two circular cylinders into three regimes based on the spacing ratio. Regime 1 , the free shear layers separating from the upstream cylinder overshoot the downstream cylinder, forming one vortex street. Regime 2, the free shear layers separated from the upstream cylinder is reattached on the downstream cylinder. A single vortex street is formed behind the downstream cylinder. 

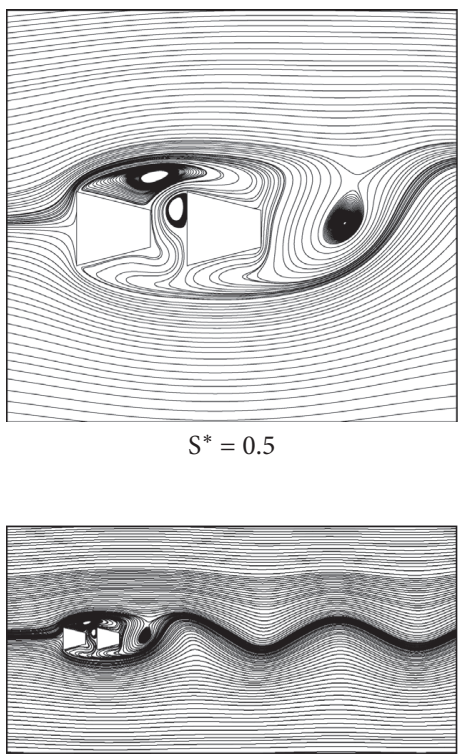

$\mathrm{S}^{*}=0.5$

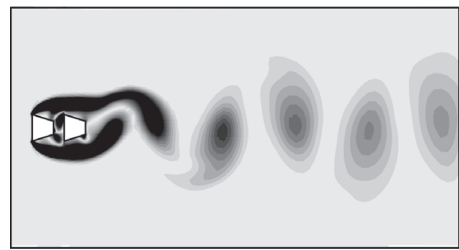

$S^{*}=0.5$

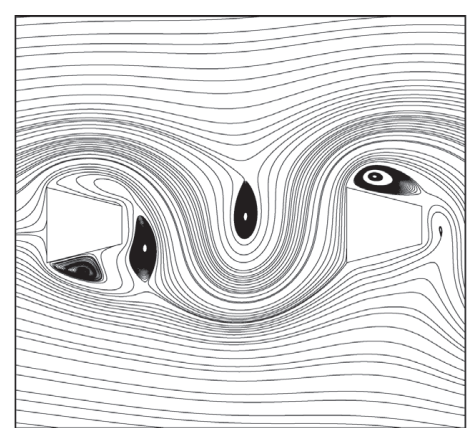

$S^{*}=3$

(a)

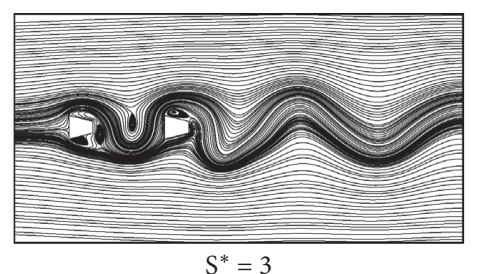

(b)

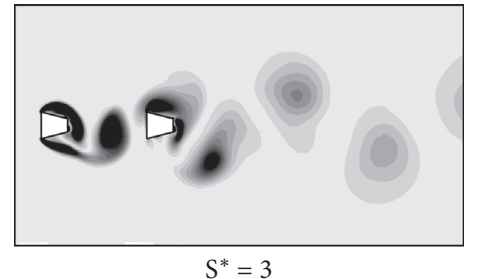

(c)
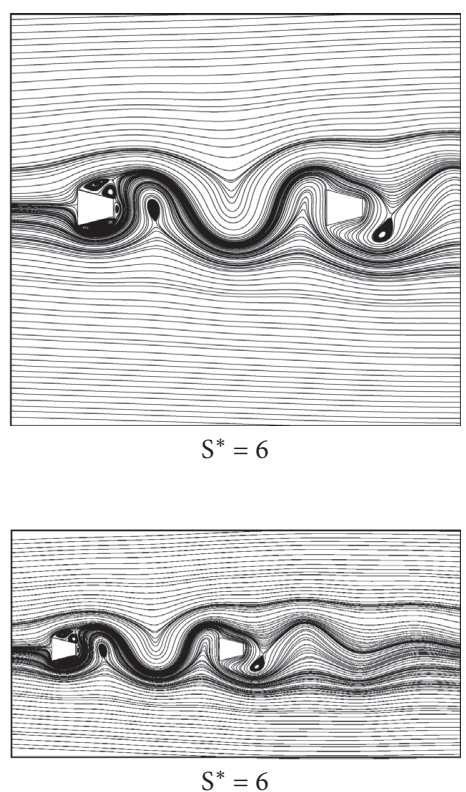

$\mathrm{S}^{*}=6$

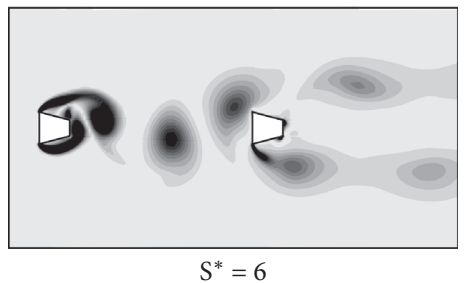

FIGURE 9: Instantaneous flow visualization images for (a) enlarged detail of instantaneous streamlines between two trapezoidal cylinders, (b) instantaneous streamlines contours, and (c) vorticity contours.

Regime 3, the free shear layers roll up alternately to form vortices between the cylinders. Vortices generate at both cylinders. Sumner [15] summarized the research studies on the flow around two tandem circular cylinders and divided the flow patterns into (i) extended-body regime; (ii) reattachment regime; (iii) co-shedding regime. The study of $\mathrm{Xu}$ and Zhou [19] found that the flow patterns around the two tandem circular cylinders is not only related to the spacing ratio but also to the Reynolds number. The change of the Reynolds number will lead to the change of the critical spacing ratio under different modes.

Similar phenomena were observed in the studies of the flow around two square cylinders in tandem by Sohankar and Etminan [16], Choi et al. [17], More et al. [20], and Yen et al. [18]. The flow pattern changes with the spacing ratio. Yen et al. [18] also divided the flow characteristics into three modes: single mode, reattached mode, and binary mode. The aerodynamic parameters of cylinders and critical spacing ratio of each mode are different from those of the circular cylinders. The most notable to the flow around two circular cylinders is that the wake of the downstream square cylinder forms a double vortex street in reattached and binary modes.

The flow around two tandem trapezoidal cylinders was similar to that around square cylinders. We divided the flow modes around two identical tandem cylinders into three categories: extended-body regime (Mode I, $S^{*} \leq 1$ ), reattachment regime (Mode II, $2 \leq S^{*} \leq 3$ ), and binary regime (Mode III, $S^{*} \geq 4$ ), as above. The characteristics and distinction of the three modes are shown in Table 2.

In mode $\mathrm{I}$, the $C_{p}$ of the windward side $(\mathrm{EF})$ of downstream trapezoidal cylinder was negative. The $C_{d \text {,avg }}$ of the upstream one was slightly smaller than that of the single trapezoidal cylinder, and the $C_{d \text {,avg }}$ of the downstream trapezoidal cylinder was negative. As the spacing ratio increased, there was a critical spacing ratio that changed the $C_{d \text { avg }}$ of the downstream trapezoidal cylinder from negative to positive (also the $C_{p}$ of the EF surface). This transition could be a demarcation between mode I and mode II. The time-average streamwise velocity between the two trapezoidal cylinders was U-shaped. There was no vortex forming between two cylinders; at the same time, there was only one vortex in the wake of the downstream trapezoidal cylinder. In mode I, the two trapezoidal cylinders behave like a single bluff-body.

In mode II, the $C_{l, \text { rms }}$ of the downstream trapezoidal cylinder was larger than that of the upstream trapezoidal cylinder. The $C_{p}$ of the EF surface reduces significantly. The time-average streamwise presents a horizontal S-shape 
TABLE 2: Characteristics and distinction of the three modes.

\begin{tabular}{|c|c|c|c|c|c|}
\hline Reference volume & Location or relationship & Mode I $S^{*} \leq 1$ & Mode II $2 \leq S^{*} \leq 3$ & Mode III $S^{*} \geq 4$ & Source \\
\hline$C_{d, \text { avg }}$ & Downstream cylinder & Negative (-) & Positive $(+)$ & Positive (+) & Figure 3(a) \\
\hline$C_{l, \mathrm{rms}}$ & $\begin{array}{c}\text { Comparison between the } \\
\text { two }\end{array}$ & $\mathrm{Up} \approx$ down & Up $>$ down & $\mathrm{Up}<$ down & Figure $3(\mathrm{~b})$ \\
\hline & $\begin{array}{l}\text { EF surface } \\
\text { FG surface }\end{array}$ & Negative $(-)$ & $\begin{array}{l}\text { Positive (+) } \\
\text { Minimum }\end{array}$ & Positive $(+)$ & $\begin{array}{l}\text { Figure } 6(b) \\
\text { Figure } 6(b)\end{array}$ \\
\hline $\begin{array}{l}\text { Time-average streamwise } \\
\text { velocity }\end{array}$ & $\begin{array}{c}\text { Center line between the } \\
\text { two }\end{array}$ & U-shaped & S-shaped $x / D=1$ & $\begin{array}{l}\text { S-shaped } \\
x / D=0.6\end{array}$ & Figure $7(\mathrm{a})$ \\
\hline Flow visualization image & The two cylinders & $D$ DE: $P=$ & $\Delta D=$ & DDO & Figure 9 \\
\hline \multicolumn{2}{|c|}{ Names of the three modes } & $\begin{array}{l}\text { Extended-body } \\
\text { regime }\end{array}$ & $\begin{array}{l}\text { Reattachment } \\
\text { regime }\end{array}$ & Binary regime & \\
\hline
\end{tabular}

Note: $x / D=0.6(x / D=1)$ was the location of the stationary point.

(Figure 7 (a)) and the stationary point was around $x / D=1$. The boundary layer separated from the upstream cylinder was reattached to the downstream cylinder.

In mode III, the distribution of the aerodynamic coefficients $\left(C_{d \text {,avg }}, C_{l, \text { rms }}\right.$, and $\left.C_{p}\right)$ were similar to that of the single one. The time-average streamwise velocity between the two trapezoidal cylinders was S-shaped, where the stationary point was around $x / D=0.6$. In this mode, the separated shear layers of the upstream cylinder are rolled up alternately in front of the downstream cylinder and form vortices. The vortex street behind the downstream cylinder is called binary flow because each vortex street is actually composed of two vortices formed behind the upstream cylinder and the downstream cylinder.

To sum up, the flow pattern around two tandem cylinders could divide into three modes according to the differences of the aerodynamic characteristics and flow field structure under different spacing ratios. Under different cross sections (circular, square, and trapezoidal), the aerodynamic parameters and critical spacing ratio of each mode were different.

\section{Conclusions}

In this paper, the numerical simulation of the flow was carried out around two identical trapezoidal cylinders in a tandem arrangement at $\operatorname{Re}=2.2 \times 10^{4}$. This study investigated the interference of flow past two tandem trapezoidal cylinders at different spacing ratios of $S^{*}=0.5-10$. The aerodynamic characteristics, wake flow, and vortex shedding patterns of the two cylinders were considered. The spacing ratio had a significant effect on the flow field around two tandem identical trapezoidal cylinders. According to the differences of the aerodynamic characteristics and flow field structure under different spacing ratios, we divided the flow modes into three categories: extended-body regime (Mode I, $S^{*} \leq 1$ ), reattachment regime (Mode II, $2 \leq S^{*} \leq 3$ ), and binary regime (Mode III, $S^{*} \geq 4$ ).

At the spacing ratio of $S^{*}=0.5,1$, the downstream cylinder was located in the trailing vortex formed by the boundary layer separated from the upstream cylinder, which led to a relatively low amplitude of the lift oscillations. The average drag coefficient of the downstream trapezoidal cylinder was negative, and the sum of drag coefficient of the two cylinders was minimum. The separated boundary layer of the upstream cylinder only formed a single-row vortex at the tail of the downstream cylinder. There was no vortex between the two cylinders. In mode I, the downstream trapezoidal cylinder acts as an extended-body of the upstream one.

When the spacing ratio was $2 \leq S^{*} \leq 3$, the flow field around the structure was in reattachment regime. The downstream cylinder was affected by the reattachment of the wake flow of the upstream cylinder. There were many smallscale vortices around the downstream cylinder, and the lift amplitude spectrum became messy and multiple low peaks appear. The maximum value of the downstream trapezoidal cylinder $C_{l, \text { rms }}$ appeared in this mode. In mode II, the boundary layer separated from the upstream cylinder and reattached on the downstream cylinder. The large negative pressure on the upper surface of the downstream cylinder was one of the important characteristics that distinguishes mode II from the other two modes.

As the spacing ratio increases to $S^{*} \geq 4$, the influence of the upstream trapezoidal cylinder on the downstream cylinder was further weakened. The aerodynamic parameters of the upstream cylinder were similar to that of the flow around a single one. Compared to mode II, the stationary point between the two trapezoidal cylinders changed from the distant vortices $(x / D \approx 1)$ to the closer vortices $(x / D \approx 0.6)$. In this mode, the separated shear layers of the upstream cylinder were rolled up alternately in front of the downstream cylinder and form vortices. Because each vortex street was actually composed of two vortices formed behind the upstream cylinder and the downstream cylinder, this mode was called binary regime.

\section{Nomenclature}

U: Upstream velocity $(\mathrm{m} / \mathrm{s})$

D: Height of the windward side of the trapezoidal cylinder

$B$ : Width of the trapezoidal cylinder

$d$ : Height of the rear end of the trapezoidal cylinder

S: $\quad$ Spacing length 
$S^{*}: \quad$ Spacing ratio

$k$ : $\quad$ Kinetic energy of turbulence

$p, \bar{p}$ : Pressure and average pressure

$F_{d}$ : Drag force

$F_{l}: \quad$ Lift force

$C_{d}$ : Drag coefficient

$C_{l}$ : Lift coefficient

$C_{p}:$ Pressure coefficient

$C_{p b}$ : Mean back pressure coefficient

$L_{r}$ : Swing length

$f: \quad$ Frequency of vortex shedding

Re: Reynolds number

St: Strouhal number

$u$ : $\quad$ Fluid velocity

$\bar{u}, u^{\prime}$ : Average and fluctuation component of velocity

$x, y$ : Cartesian coordinates

$v$ : $\quad$ Fluid kinematic viscosity

Greek letters

$\rho$ : Fluid density

$\varepsilon$ : Rate of dissipation of turbulent kinetic energy

$\mu$ : Fluid dynamic viscosity

Subscripts

avg: Time averaged

rms: Root mean square

$i, j$ : Component in $x$ and $y$ directions.

\section{Data Availability}

The data used to support the findings of this study are available from the corresponding author upon request.

\section{Conflicts of Interest}

The authors declare that there are no conflicts of interest regarding the publication of this paper.

\section{Acknowledgments}

This work was supported by the National Natural Science Foundation of China (NSFC), project no. 51675450.

\section{References}

[1] S. C. Luo, M. G. Yazdani, Y. T. Chew, and T. S. Lee, "Effects of incidence and afterbody shape on flow past bluff cylinders," Journal of Wind Engineering \& Industrial Aerodynamics, vol. 53, no. 7, pp. 375-399, 1994.

[2] A. Venugopal, A. Agrawal, and S. V. Prabhu, "Influence of blockage and upstream disturbances on the performance of a vortex flowmeter with a trapezoidal bluff body," Measurement, vol. 43, no. 4, pp. 603-616, 2010.

[3] T. S. Lee, "Early stages of an impulsively started unsteady flow past non-rectangular prisms," Computers \& Fluids, vol. 27, no. 4, pp. 435-453, 1998.

[4] R. Kahawita and P. Wang, "Numerical simulation of the wake flow behind trapezoidal bluff bodies," Computers \& Fluids, vol. 31, no. 1, pp. 99-112, 2002.

[5] M. Cheng and G. R. Liu, "Effects of afterbody shape on flow around prismatic cylinders," Journal of Wind Engineering and Industrial Aerodynamics, vol. 84, no. 2, pp. 181-196, 2000.
[6] Y. J. Chung and S.-H. Kang, "Laminar vortex shedding from a trapezoidal cylinder with different height ratios," Physics of Fluids, vol. 12, no. 5, pp. 1251-1254, 2000.

[7] A. Dhiman and M. Hasan, "Flow and heat transfer over a trapezoidal cylinder: steady and unsteady regimes," AsiaPacific Journal of Chemical Engineering, vol. 8, no. 3, pp. 433-446, 2013.

[8] L. Ding, L. Zhang, and D. Y. Jiang, "Flow induced motion of bluff bodies with different cross sections in flow field with high Reynolds number," Journal of Vibration and Shock, vol. 34, no. 12, pp. 176-181, 2015.

[9] J. J. Miau, J. T. Wang, J. H. Chou, and C. Y. Wei, "Lowfrequency fluctuations in the near-wake region of a trapezoidal cylinder with low aspect ratio," Journal of Fluids and Structures, vol. 17, no. 5, pp. 701-715, 2003.

[10] M. M. Zdravkovich, "The effects of interference between circular cylinders in cross flow," Journal of Fluids and Structures, vol. 1, no. 2, pp. 239-261, 1987.

[11] M. M. Zdravkovich, "Review-review of flow interference between two circular cylinders in various arrangements," Journal of Fluids Engineering, vol. 99, no. 4, pp. 618-633, 1977.

[12] M. M. Zdravkovich, "Smoke observations of wakes of tandem cylinders at low Reynolds numbers," Aeronautical Journal, vol. 76, no. 734, pp. 108-114, 1972.

[13] J. R. Meneghini, F. Saltara, C. L. R. Siqueira, and J. A. Ferrari, "Numerical simulation of flow interference between two circular cylinders in tandem and side-by-side arrangements," Journal of Fluids and Structures, vol. 15, no. 2, pp. 327-350, 2001.

[14] R. J. Jiang, J. Z. Lin, and X. K. Ku, "Flow-induced vibrations of two tandem circular cylinders in a parallel-wall channel," Physics of Fluids, vol. 26, no. 10, p. 413, 2014.

[15] D. Sumner, "Two circular cylinders in cross-flow: a review," Journal of Fluids and Structures, vol. 26, no. 6, pp. 849-899, 2010.

[16] A. Sohankar and A. Etminan, "Forced-convection heat transfer from tandem square cylinders in cross flow at low Reynolds numbers," International Journal for Numerical Methods in Fluids, vol. 60, no. 7, pp. 733-751, 2009.

[17] C. B. Choi, Y. J. Jang, and K. S. Yang, "Secondary instability in the near-wake past two tandem square cylinders," Physics of Fluids, vol. 24, no. 2, pp. 1-22, 2012.

[18] S. C. Yen, K. C. San, and T. H. Chuang, "Interactions of tandem square cylinders at low Reynolds numbers," Experimental Thermal and Fluid Science, vol. 32, no. 4, pp. 927-938, 2008.

[19] G. Xu and Y. Zhou, "Strouhal numbers in the wake of two inline cylinders," Experiments in Fluids, vol. 37, no. 2, pp. 248-256, 2004.

[20] B. S. More, S. Dutta, M. K. Chauhan, and B. K. Gandhi, "Experimental investigation of flow field behind two tandem square cylinders with oscillating upstream cylinder," Experimental Thermal and Fluid Science, vol. 68, pp. 339-358, 2015.

[21] J. O. Hinze, Turbulence, McGraw-Hill Publishing Co., New York, USA, 1975.

[22] T.-H. Shih, W. W. Liou, A. Shabbir, Z. Yang, and J. Zhu, “A new $k-\varepsilon$ eddy viscosity model for high Reynolds number turbulent flows," Computers \& Fluids, vol. 24, no. 3, pp. 227-238, 1995.

[23] D. A. Lyn, S. Einav, W. Rodi, and J.-H. Park, "A laser-Doppler velocimetry study of ensemble-averaged characteristics of the turbulent near wake of a square cylinder," Journal of Fluid Mechanics, vol. 304, pp. 285-319, 1995. 
[24] C. Norberg, "Flow around rectangular cylinders: pressure forces and wake frequencies," Journal of Wind Engineering and Industrial Aerodynamics, vol. 49, no. 1-3, pp. 187-196, 1993.

[25] A. Sohankar, "A numerical investigation of the flow over a pair of identical square cylinders in a tandem arrangement," International Journal for Numerical Methods in Fluids, vol. 70, no. 10, pp. 1244-1257, 2012.

[26] Y. Srinivas, G. Biswas, A. S. Parihar, and R. Ranjan, "Largeeddy simulation of high Reynolds number turbulent flow past a square cylinder," Journal of Engineering Mechanics, vol. 132, no. 3, pp. 327-335, 2006.

[27] Q. Zhou, H. L. Liao, and S. Y. Cao, "Numerical study of flow characteristic around square cylinder at high Reynolds number," Journal of Southwest Jiaotong University, vol. 53, no. 3 , pp. $533-539,2018$.

[28] Atsushi and Okajima, "Strouhal numbers of rectangular cylinders," Journal of Fluid Mechanics, vol. 123, pp. 379-398, 1982.

[29] X. K. Wang, Z. Hao, J. X. Zhang, and S. K. Tan, "Flow around two tandem square cylinders near a plane wall," Experiments in Fluids, vol. 55, no. 10, pp. 1-14, 2014.

[30] M. K. Kim, D. K. Kim, S. H. Yoon, and D. H. Lee, "Measurements of the flow fields around two square cylinders in a tandem arrangement," Journal of Mechanical Science and Technology, vol. 22, no. 2, pp. 397-407, 2008.

[31] W. Zhang, H.-S. Dou, Z. Zhu, and Y. Li, "Unsteady characteristics of low-Re flow past two tandem cylinders," Theoretical and Computational Fluid Dynamics, vol. 32, no. 4, pp. 475-493, 2018. 